\title{
Inductively coupled power transfer (ICPT) for electric vehicle charging - A review
}

Kafeel Ahmed Kalwar, Muhammad Aamir, Saad Mekhilef*

Power Electronics and Renewable Energy Research Laboratory (PEARL), Department of Electrical Engineering, University of Malaya, Kuala Lumpur, 50603, Malcysia

A R T I C L E I N F O

Article history:

Received 26 July 2014

Received in revised form

12 January 2015

Accepted 8 March 2015

Available online 30 March 2015

Keywords:

Electric vehicle (EV)

Wireless charging of electric vehicle

Inductively coupled power transfer (ICPT)

\begin{abstract}
A B S T R A C T
The deficiency in the availability of petroleum products has given rise to the incorporation of electric vehicles (EVs) globally as a substitute for the conventional transportation system. Significant research has been pursued over last two decades in the development of efficient EV charging methods. A preliminary review of few methods developed for wireless charging revealed that ICPT is a promising and convenient method for the wireless charging of EVs. This paper includes the equivalent circuit analysis and characteristics of the ICPT system and focuses on the research progress in respect of the designs for the charging coil, leakage inductance compensation topologies, power level enhancement and misalignment toleration. The improvement in these factors has been essential for the implementation of EV charging. A brief discussion over design process and control of ICPT system has been added. Conclusions have been made on the basis of the information extracted from the literature and some future recommendations are provided.
\end{abstract}

c 2015 Elsevier Ltd. All rights reserved.

Contents

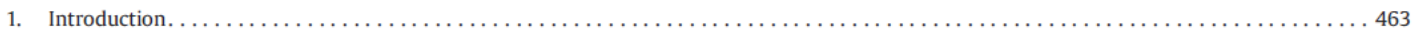

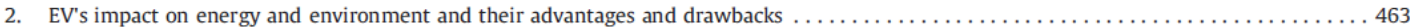

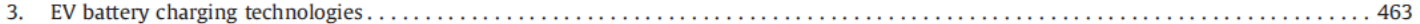

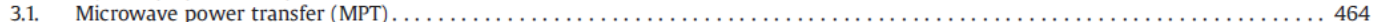

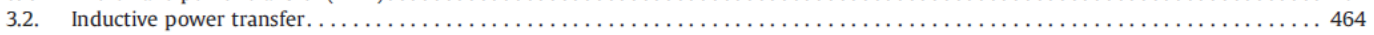

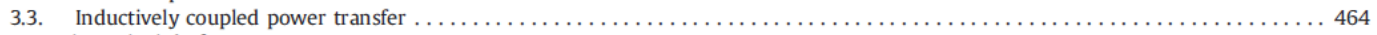

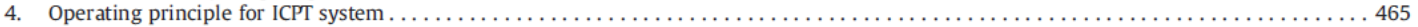

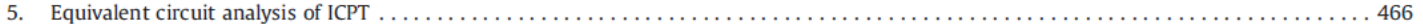

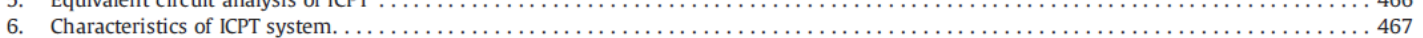

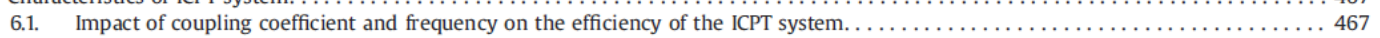

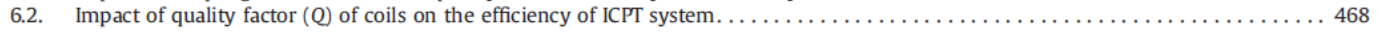

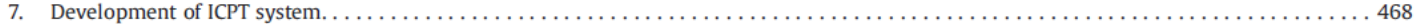

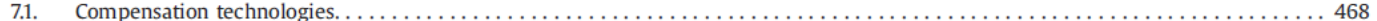

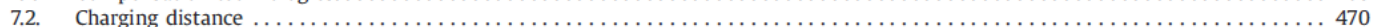

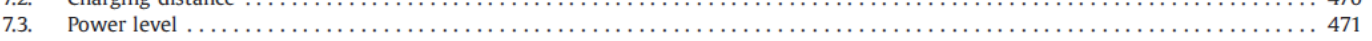

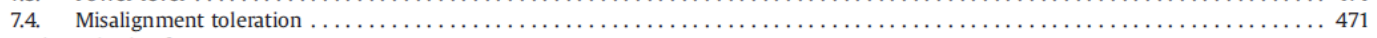

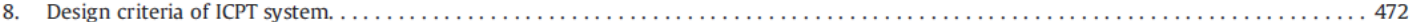

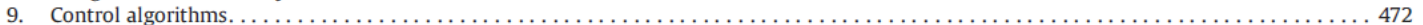

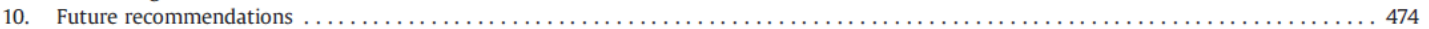

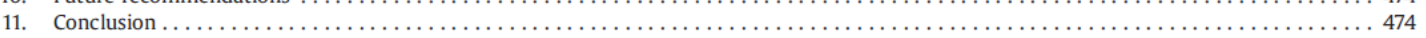

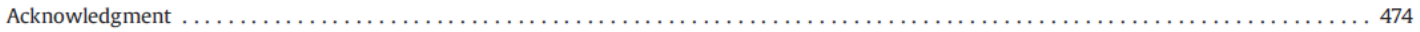

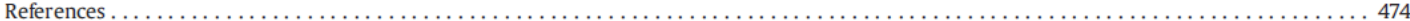

\footnotetext{
* Corresponding author. Tel.: +60379677611 .

E-mail address: saad@um.edu.my (S. Mekhilef).
}

http://dx.doi.org/10.1016/j.rser.2015.03.040

1364-0321/० 2015 Elsevier Ltd. All rights reserved. 


\section{Introduction}

Petroleum products, including compressed natural gas and liquefied petroleum gas, have remained the main resources of the energy demand-supply cycle for transportation [1]. The gradual increase in energy demand has created a scarcity of these naturally available resources. Moreover, carbon dioxide emission contributing global warming and high fuel prices have compelled scientists and researchers to find a sustainable alternate solution to these depleting natural energy reserves [2-4]. Substantial research and development resources are being devoted towards alternate energy technologies [5-7].

The incorporation of electric vehicles (EVs) has been considered to be a suitable replacement for the depleting petroleum resources used for automotive applications $[8,9]$. The concept of EV was developed with the advent of the Hybrid Electric Vehicle (HEV), which later led to the introduction of plug-in hybrid electric vehicles (PHEVs). But the PHEV exhibited several disadvantages such as such the need of connecting cable and plug in charger, galvanic isolation of on-board electronics, the size and weight of the charger, and more importantly safety issues concerning their operation in the rain and snow [10]. In order to incorporate the aforementioned disadvantages in PHEV, pure EV has been developed $[11,12]$. Electric vehicles (EV) enable smoke free transportation, and offer an effective solution to the adverse environmental impacts of the conventional transportation system [13], and are powered over a large air gap through wireless charging even in bad weather and harsh environmental conditions without utilizing plugs or wires [14]. However the EVs are operated by means of electrical energy, so these are polluting the environment indirectly depending on the source of energy. They operate with low emissions of greenhouse gases [15]. Furthermore, they can be used with smart house systems to supplement the energy storage through bidirectional energy transfer [16]. Although EV is a possible substitute for depleting energy reserves and to overcome the environmental issues, the limited mileage and time needed to recharge, less misalignment toleration and overall cost involved are still limitations. These issues require special consideration for the successful implementation of an electrically driven vehicle system worldwide. The quest for a solution to the aforesaid issues has led researchers to develop the most useful method of wireless charging of EVs. Several methods have been pursued to charge both stationary and moving electric vehicles $[17,18]$. Static charging of EVs is convenient for the users to utilize at home or office parking [19], while dynamic charging allows powering the moving EVs. Though the static charging of EVs has the advantage of low cost compared to dynamic charging, but these require heavy size batteries so to store more charge [10], while the dynamic charging makes use of several charging coils paved in the roadbed at a spaced distance which allows more vehicles to get charged simultaneously [20]. Thus the dynamic charging has the main advantage of avoiding the need for a high capacity battery and reduces the battery cost. Inductively coupled power transfer (ICPT) has been most successful in consideration for the wireless charging of EV. Due to reasonable electrical isolation between transmitting coil and receiving coil, the ICPT system has ability to operate in grimy conditions. It is highly reliable and requires less maintenance. Extensive research over the span of time, in different technical aspects, has improved the performance of ICPT in terms of charging distance, misalignment toleration, high power and high efficiency $[21,22]$.

This paper reviews the methods developed for the wireless charging of electric vehicles and their deployment as well as the evolution of ICPT performance. It begins with the impact of electric vehicles on energy and the environment and a brief comparison of the different EV charging methods in Sections 2 and 3 respectively. The operational mechanism of the ICPT method is discussed in Section 4. The equivalent circuit analysis and characteristics of the ICPT system are provided in Sections 5 and 6. A discussion on the research progress of ICPT is presented in Section 7. The discussion on design criteria and control algorithm is presented in Sections 8 and 9 respectively. Few recommendations and a conclusion based on the information learnt from the literature are provided in Sections 10 and 11 .

\section{EV's impact on energy and environment and their advantages and drawbacks}

Transportation causes the consumption of $27 \%$ world petrolatum energy and 33.7\% of greenhouse gas emissions in $2012[1,8]$. To reduce the $\mathrm{CO}_{2}$ emissions, legislation is being introduced in some countries to limit the emissions from cars and other means. Substantial use of natural resources has created a scarcity thereof, and, in future, the world may face the impact of the dependence on these depleting natural energy resources.

Electricity has proven to be a viable alternative transportation fuel to replace petroleum products, so the electric vehicle has paved the path for reducing the dependence on petroleum energy. The EVs are solely operated by electric power and generates zero emissions of greenhouse gases. This creates substantial changes to reduce the air pollution, thus making the populated surroundings cleaner and gratifying for all livings around. The noise of conventional internal combustion engines is avoided through adoption of EV and the metropolises becomes noiseless [23].

With the development of wireless charging of EVs, the users do not need any wires to charge them safely. With automatic charging, the users simply need to park the EV over the plugless charging pad. Through the development in technology, the dynamic charging of electric vehicles has been introduced, which enables the charging of moving vehicles on the roadway. Regular stops on the roadway offer charging points to avoid disrupting the timetable of users. The charging system can operate reliably even in the most adverse weather conditions of snow and rain.

Particularly, the electric vehicles can be connected to the smart grid of the future. The batteries of EVs have the potential to benefit both consumers and the overall electrical grid [24] and by feeding electricity back to the grid during peak demand it helps to keep the overall electricity cost stable. In addition, the charged vehicle could also potentially serve as an emergency supply for home or business in case of power outage.

There is risk of safety during accidents in case of human or animal presence under the car or bus. Thus, there is a major concern of electromagnetic field exposure in wireless charging of electric vehicles. The EMI exposure has to be under limited range as prescribed safety regulatory authorities during normal as well as abnormal operation.

The main drawback of EV is requirement of a battery of high energy density. It corresponds to frequent mileage of electric vehicle. More the mileage, larger the battery pack will be used. Lead acid battery is mostly used, which is very expensive. Escalating demand of EVs will result in the deficit of energy gradually introducing load management issues in grids.

The provoked concern of environment pollution and energy issues will rapidly ensure the feasibility of EVs for common commercialization by manufacturing light weight high energy density batteries, technical advancements to overcome misalignment, low efficiency during dynamic behavior of the power transfer system.

\section{EV battery charging technologies}

Different EV battery charging methods have been developed in the quest to achieve high efficiency, large power transfer and other attributes. The deployed methods have approached the different 
capabilities in terms of power level, air gap and efficiency. These methods are discussed in this section.

\subsection{Microwave power transfer (MPT)}

This technology enables the transmission of power and information by using radio waves whose wavelength range falls into the category of microwaves. The operation of MPT involves components including a microwave generator, transmitting antenna and receiving antenna (also termed as rectenna). The block diagram shows the mechanism of MPT. Initially, the power supplied from the $50 \mathrm{~Hz}$ grid is converted into DC, which is fed into a microwave generator. There are resonating cavities in the microwave generator through which the current passes and produces microwave electromagnetic radiation. The rectenna receives the microwave energy and converts it back to DC. The received DC is utilized as per the requirements of the application.

Kyoto University has been working on the implementation of the MPT for the charging of EV for some years. In 2000, Kyoto University developed a MPT system for EV charging and achieved an efficiency of $76 \%$ [25]. A $10 \mathrm{~kW}$ rectenna array capable of receiving $3.2 \mathrm{~kW} / \mathrm{m}^{2}$ at a circa distance of $4 \mathrm{~m}$ with efficiency of $84 \%$ has been developed by the Volvo Technologies Japan and the Nihon Dengyo Kosaku companies [26]. Although the MPT has the advantage of transferring power over longer distances, it has the disadvantage of increased cost and antenna size. In addition, high power transmission using microwaves is not considered safe for humans as it does not comply with the radio wave regulation for high power. To overcome the disadvantage of the cost and antenna size, a new technique "Microwave Building" was introduced in [27]. In this technique, a waveguide is used, which provides a path for microwaves and does not allow the diffusion of microwaves. Therefore, the size of antenna and cost are reduced to a great extent and the technique is considered safe. However, to date, low power has been transferred using this technique.

\subsection{Inductive power transfer}

The charging of plugin electric vehicles has always a safety risk of the direct contact of metal-to-metal. To avoid this concern, the designs of electric vehicle charging systems were developed based on inductive power transfer (IPT) [28]. The IPT works on electromagnetic induction phenomena to transfer power through an ai cored transformer with closely spaced primary and secondary coils $[2,14,29]$. The coils seem to be connected physically to each other but are isolated electrically, as shown in Fig. 1(a). The extended picture in Fig. 1(b) shows a sealed charging pad. The charger is inserted into the vehicle like fueling a conventional vehicle. The schematic diagram of IPT is shown in Fig. 2

Inductive power transfer has been successfully implemented for EV battery charging. This method showed promising high power transfer with a smaller air gap, however, when the air gap between the primary and secondary coils is increased the performance decreases drastically due to leakage inductance [29].

\subsection{Inductively coupled power transfer}

The inductive power transfer offers low efficiency when the air gap is increased between charging coils and also involves wired chargers, while the designs for full wireless charging systems have been developed to overcome the deficiencies of IPT and make the charging system convenient for the users. The inductively coupled power transfer (ICPT) employs capacitors connected to both the primary and secondary coils to compensate the leakage flux due to the increased air gap, as shown in Fig. 3. Both the LC circuits work on resonance phenomena to enable effective energy transfer at resonant frequency [31].

The inductively coupled power transfer (ICPT) method is known worldwide for its high power transfer in many applications, mainly in electric vehicles [5,31]. It provides a rapid charging process, and optimized power transmission by frequency variation

b
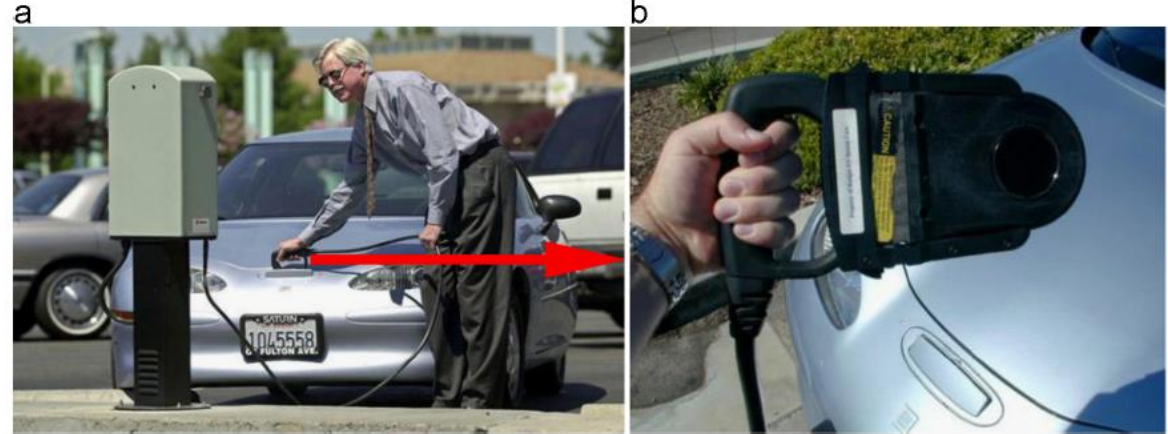

Fig. 1. (a) EV charging through inductive power transfer [30], (b) charging pad [30].

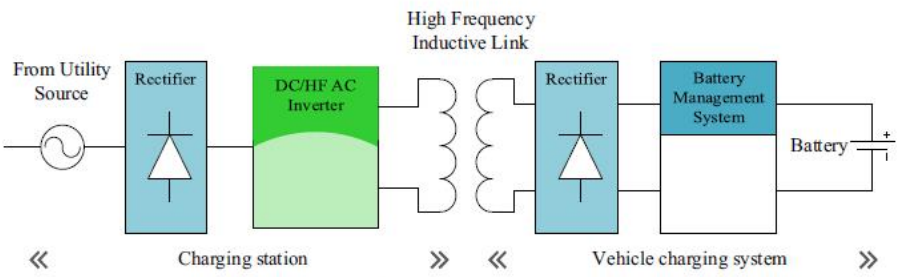

Fig. 2. Schematic diagram of Inductive Power Transfer. 
and control over the loss due to low magnetic coupling. The ICPT has been used for both stationary and moving electric vehicles [21]. If the vehicle being charged is stationary, it is said to be "charging of the battery electric vehicle (BEV)" or "static charging", while, if the vehicle is moving, some names given to it are dynamic charging and online electric vehicle (OLEV). In the OLEV set-up, the primary coil is placed in the pavement at spaced locations, thereby establishing a charging roadbed that allows power transfer at several spaced locations throughout the roadbed. Although it involves high capital cost for infrastructure installation, its feature of frequent charging can elude the need for high capacity batteries. The online electric vehicle (OLEV) uses relatively low resonant frequency as the system is installed for the charging of moving electric vehicles in an open public area. Therefore, it is necessary to maintain safety regulations prescribed by safety regulation organizations, such as the International Committee on Electromagnetic Safety (ICES), and the International Commission on Non-ionizing

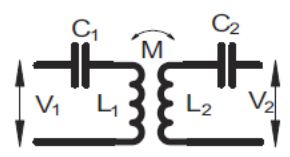

Fig. 3. ICPT circuit.

Table 1

ICPT applied projects.

\begin{tabular}{lllc}
\hline Manufacturer/institute & Power rating & Efficiency (\%) & Air gap (cm) \\
\hline $\mathbf{2 0 0 7}[32]$ & $60 \mathrm{~W}$ & 40 & 200 \\
$\mathbf{2 0 1 1}[37]$ & $1 \mathrm{~kW}$ & 88 & 30 \\
$\mathbf{2 0 1 1}[38]$ & $3.3 \mathrm{~kW}$ & $84-90$ & 10 \\
$\mathbf{2 0 1 1}[39]$ & $60 \mathrm{~kW}$ & 92 & 40 \\
$\mathbf{2 0 1 2}[40]$ & $3.3 \mathrm{~kW}$ & 90 & 18 \\
$\mathbf{2 0 1 2}[26]$ & $30 \mathrm{~kW}$ & 92 & 14 \\
$\mathbf{2 0 1 3}[41]$ & $180 \mathrm{~kW}$ & 85 & 10 \\
$\mathbf{2 0 1 4}[42]$ & $300-600 \mathrm{~kW}$ & 83 & 3 \\
\hline
\end{tabular}

Radiation Protection (ICNIRP) to avoid the exposure by humans to the electromagnetic field (EMF).

Many authors have termed this technology as the inductively coupled power transfer (ICPT), while Kurs et al. [32], and Karalis et al. [33] termed this method as the strong magnetic coupled power transfer. Nevertheless, both of these indicate same characteristics $[13,34]$. Takanashi et al. [35] split IPT based on the use of the magnetic core and operating frequency into the inductively coupled power transfer (ICPT ) method [31] and the magnetic resonant power transfer method $[32,33]$. According to [35], the ICPT works on a frequency of less than $200 \mathrm{kHz}$ and employs a ferrite core, while the magnetic resonance power transfer works on a frequency higher than $1 \mathrm{MHz}$ and does not utilize a ferrite core.

The literature suggests that the ICPT is a comparatively efficient, optimum and light charging system through which power can be transferred from one part to another part of the system with no physical contacts, particularly in the electric vehicle application, even in extreme weather conditions [13]. Inductive power transfer (ICPT) has been attracting the interest of manufacturers in the last few years [36]. Cellular phone charging through ICPT is an application that has already been commercialized. The common commercialization of this technology is likely to be unveiled soon and may modernize the automotive industry.

The achievements in research of ICPT technology are tabulated in Table 1:

A comparison of charging methods with respect to the parameters including efficiency, frequency, cost, power level and distance is shown in Table 2.

From developed methods of electric vehicle charging, the ICPT method has enabled the high power transfer.

\section{Operating principle for ICPT system}

In the ICPT system, unlike the transformers in which the primary and secondary windings are wound on a magnetic core to avoid the leakage of inductance, and to produce a good coupling, the windings

Table 2

Comparison of various EV battery charging methods.

\begin{tabular}{|c|c|c|c|c|c|}
\hline Microwave power transfer & Medium & $1-30 \mathrm{GHz}$ & Low/medium & Long & High \\
\hline Inductive power transfer (Uncompensated) & High & $15-100 \mathrm{kHz}$ & High & Very low & High \\
\hline
\end{tabular}

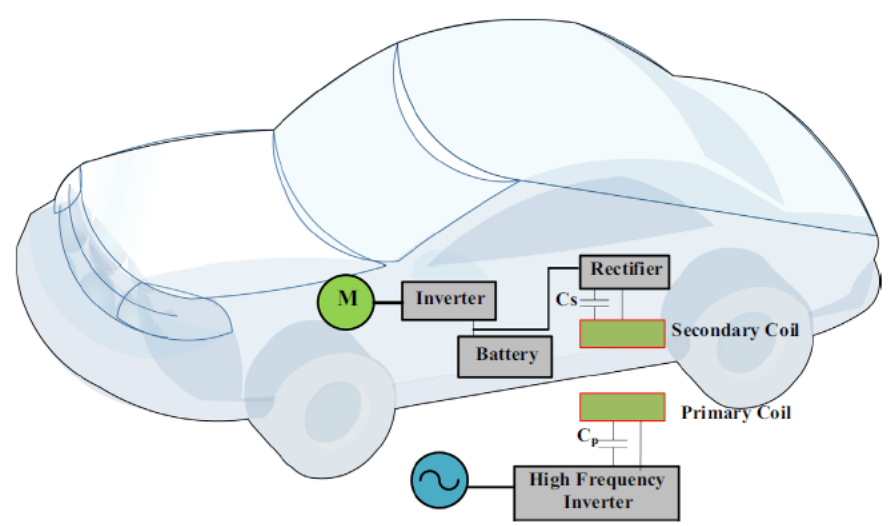

Fig. 4. Components of wireless power transfer for EV charging. 
are not linked through a common magnetic core but separated over a large air gap. The inductively coupled power transfer for the EV charging system is depicted in Fig. 4, which consists of a utility supply, high-frequency inverter, primary and secondary resonant circuits and EV load [22]. The primary coil in Fig. 4 is a charging pad that is fixed in the ground. The EV is parked above the charging pad and a highfrequency AC voltage is applied to the charging pad. The rate of current flow creates a time variant magnetic field around the primary coil Subsequently, by Ampere's Law and Faraday's law, being in the vicinity of a variant magnetic field, a voltage is induced at the terminals of the secondary coil, as shown in Fig. 5 [43]. The electrical load of EV is connected to the secondary coil, which completes the circuit and enables the power transfer.

As the primary and secondary coils are separated by a large air gap that results in low mutual inductance and the voltage across the mutual inductance becomes less [31], which creates a delay between the current and voltage and thus the reactive power is produced. To overcome the low power factor due to the reactive power [44], capacitance is added to both the primary and secondary coils to achieve the resonance phenomenon. This strengthens the coupling between the loosely coupled coils. The schematic diagram of the series-series compensated circuit topology is as shown in Fig. 6. This ensures the high power transfer when both the primary and secondary circuits are operated at the same resonant frequency [20,31].

The power output of an ICPT system $\left(P_{\text {out }}\right)$ can be analyzed by using two observations in the ICPT system [46]:

Open-circuit test

$V_{O C}=j \omega M I_{1}$

Short-circuit test

$I_{S C}=\frac{M I_{1}}{L_{2}}$

Then power can be calculated as the product of open circuit test voltage and short circuit test current:

$$
S_{u}=j \omega M I_{1} \times \frac{M I_{1}}{L_{2}}=\frac{j \omega M^{2} I_{1}{ }^{2}}{L_{2}}
$$

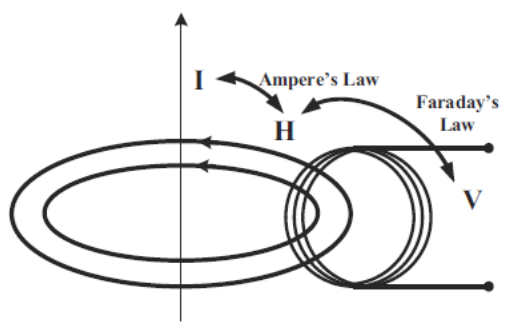

Fig. 5. Basic concept of inductive power transfer [45].
$P=S_{u} \times Q=\frac{j \omega M^{2} I_{1}{ }^{2}}{L_{2}} \times Q_{2}$

$P=\frac{j \omega M^{2} I_{1}^{2}}{L_{2}} \times \frac{\omega L_{2}}{R_{L}} ;$ Since $Q_{2}=\frac{\omega L_{2}}{R_{L}}$

Therefore, the power transferred at the secondary coil can be calculated using the following equation:

$P=\frac{\omega^{2} M^{2} I_{1}^{2}}{R_{L}}$

where $\omega$ is the angular frequency of the transmitting coil, $I_{1}$ is the current through transmitting coil, $M$ is the mutual inductance between two coils and $R_{L}$ is the load resistance.

\section{Equivalent circuit analysis of ICPT}

Although the inductively coupled power system has many configurations to compensate for the effect of leakage flux, a series-series resonant structure of the ICPT system is selected for analysis because of its simplicity and moreover it reduces the parameters that are involved in the optimization of efficiency of the ICPT system. An ICPT system with a series resonant capacitor is shown in Fig. 7. $L_{p}, L_{s}$ are the self-inductances of the primary and secondary side and $M$ is mutual inductance. $C_{p}, C_{s}$ are the capacitances of the primary and secondary side capacitors. $R_{1}, R_{2}$ are the resistances of the primary and secondary side and $R_{L}$ is the load resistance. Fig. 8 shows the T-type of equivalent circuit of the ICPT system circuit shown in Fig. 7.

The coupling coefficient $(k)$, that defines the extent of coupling of the primary and secondary coil, is given by:

$$
k=\frac{M}{\sqrt{L_{p} L_{S}}}
$$

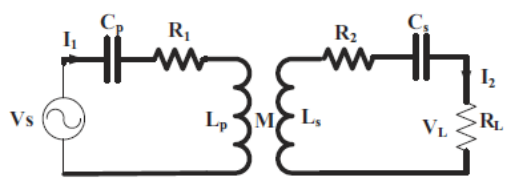

Fig. 7. Series-series (SS) resonant ICPT system.

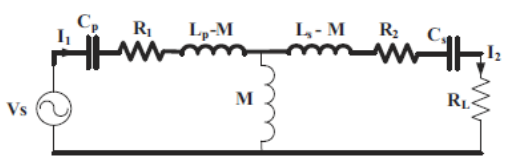

Fig. 8. Equivalent circuit of ICPT system

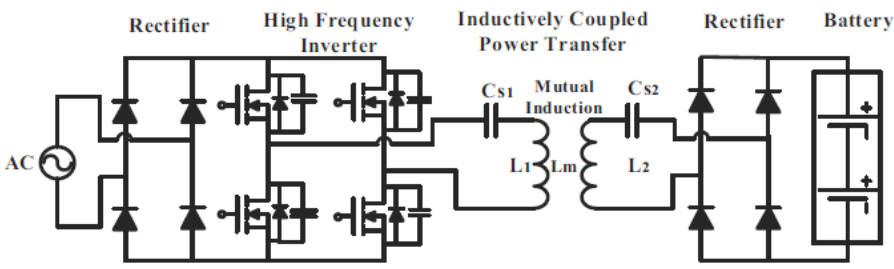

Fig. 6. Circuit of inductively coupled power transfer.

Link to full text articles :

http://www.sciencedirect.com/science/article/pii/S1364032115001938

https://ideas.repec.org/a/eee/rensus/v47y2015icp462-475.html 
http://umexpert.um.edu.my/file/publication/00005361_119906.pdf 